\title{
Research on the Relationship between Air Transport Capacity Upgrading and Economic Growth from the Perspective of Externality -Take Guizhou Province for Example
}

\author{
LV Zheng ${ }^{1, a,{ }^{*}, \text { BAI Wan-ping }}{ }^{2, b}$ \\ ${ }^{1}$ School of Mathematics and Statistics, Guizhou University of Finance and Economics, Guiyang, \\ Guizhou, China \\ ${ }^{2}$ Guizhou Statistical Application Institute, Guizhou University of Finance and Economics, Guiyang, \\ Guizhou, China \\ alvzheng151012@163.com, bbaiwp2002@sina.com \\ *LV Zheng
}

Keywords: Externality, Air transport capacity, Economic growth, VAR model, Generalized impulse response.

\begin{abstract}
How is the dependence on air transport in the post-development area? Does it exist and what spillover effects exist? It is not enough in theory and experience. On the basis of reviewing the theoretical relationship between transportation infrastructure and regional economic growth, taking air traffic volume and regional GDP in Guizhou province from 1998 to 2017 as the research object, VAR model is applied to analyze the linkage mechanism between the two. The research shows, whether it is the absolute effect of generalized impulse response or the relative effect of variance decomposition, the promotion of air transport capacity to economic growth is far more important than economic growth to air transport, and the improvement of Guizhou's air transport capacity has a negative externality to regional economic growth in the short term; the effect of economic growth on aviation is not obvious, it shows that Guizhou economy in the economic take-off stage is less dependent on air transport, but the dependence on follow-up development will gradually increase.
\end{abstract}

\section{外部性视角下航空运输能力提升与经济增长的关系研究 \\ 一以贵州省为例

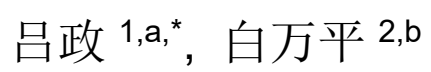 \\ 1 贵州财经大学数统学院, 贵阳, 贵州, 中国 \\ 2 贵州财经大学贵州统计应用研究院, 贵阳, 贵州, 中国 \\ alvzheng151012@163.com, bbaiwp2002@sina.com \\ *吕政}

关键词：外部性；航空运输能力；经济增长; VAR 模型；广义脉冲响应

中文摘要. 后发地区对航空运输的依赖性如何? 是否存在以及存在何种外部性?理论和经验上 的研究并不充分。在回顾交通基础设施与区域经济增长理论关系的基础上，以贵州 1998 年至 2017 年的航空运输量与地区生产总值为研究对象, 应用 VAR 模型实证分析两者之间的联动 机制。结果显示: 无论从广义脉冲响应的绝对效果还是从方差分解的相对效果判断, 航空运 输能力提升对经济增长的推动作用远大于经济增长对航空运输的拉动作用, 短期内贵州航空 运输能力提升对地区经济增长存在一定程度的负外部性; 经济增长对航空业的带动效应并不 
明显，说明处在经济起飞阶段的贵州经济对航空运输的依赖性还较弱。

\section{1. 引言}

民航舒适便利且运价较高, 作为一种高档消费品, 其需求收入弹性高于公路、铁路和水 路等一般交通运输商品, 显然居民收入水平提高将更快地扩大民航运输需求。2016 年, 《中 国民航 “十三五” 规划》提出运输总周转量和旅客运输量等主要目标的年均增速分别预期达 到 $10.8 \% 、 10.4 \%$, 明显高于 $6.5 \%$ 的全国经济增长指标, 这既是航空业发展的客观要求, 也 是尊重经济规律的反映。同年，贵州颁布的《综合交通运输 “十三五” 发展规划》指出，要 将龙洞堡机场建设成为西南地区重要的枢纽机场之一，加速建成贵州 “一枢十六支”民航机 场格局, 并提出了高于地区经济增长的奋斗目标。诚然, 一个地区的经济发展离不开交通运 输等基础设施的改善，但便利的交通也可能将当地的要素禀赋转移到其他地区。因此，以贵 州作为样本，厘清欠发达地区航空运输能力提升与经济增长的关系，对于推进同步小康和实 现中等发达国家水平的目标, 丰富发展经济学的理论, 都有着十分重要的理论和现实意义。

自 2000 年以来我国在交通运输方面的投资不断增加，张军等(2007)解释了“中国奇迹” 的实现得益于超前的交通基础设施建设 ${ }^{[1]}$, 关兴良等(2014)认为交通运输体系的进一步完善有 助于缩小地区发展差距 ${ }^{[2]}$, 吴继贵和叶阿忠(2015)研究发现经济增长促进了交通运输业发展 [3]，国内学者的研究说明我国交通运输投资与经济增长之间具有双向作用关系。民航凭借其 能够实现全球范围运输的能力, 在国民经济和社会发展中的地位越来越重要, ATAG(2008)的 报告曾指出航空运输对经济增长的贡献占据 GDP 的 $8 \%{ }^{[4]}$ 。刘兰娟和董万好(2009)以及管驰明 和马奇骐(2010)讨论了我国民航业与 GDP 之间的关系，认为民航业通过积累资本、吸引投资 和促进需求等方式带动经济增长, 但是不同时滞期的影响程度各异 ${ }^{[5-6]}$ 。卢丽娟和李晓津(2016) 的结果显示, 民航发展对经济增长的推动作用愈发显著, 民航业对服务业、批发零售业以及 金融业等有重要影响 ${ }^{[7]}$ 。绝大多数研究表明, 我国民航业发展对经济增长起到了正向的促进 作用。目前学界对航空业的研究主要集中在航空经济、临空经济以及航空物流这三个方面。 张占仓等(2016)认为临空经济表达了新的经济形态与机场的依托服务关系，这种关系改变了 地区的要素禀赋, 促进了就业增长和区域税收 ${ }^{[8]}$ 。

综上所述, 航空业发展是经济增长的助推器, 不同地区航空运输业与经济增长的作用机 制存在一定差异，但以上文献均未以外部性为视角，结合外部性能够在一定程度上解释不同 地区航空运输能力与经济增长关系存在差异的原因。本研究在对欠发达地区交通基础设施的 外部性进行理论分析的基础上, 以贵州 1998 2017 年航空运输量、地区生产总值的季度数据 为依据, 应用 VAR 模型实证航空运输能力与经济增长的联动机制。可能的边际贡献主要在两 个方面: (1)本文首先基于外部性的视角, 将贵州作为欠发达地区的代表, 探讨了欠发达地区 航空运输能力提升与经济增长之间可能存在的关系; (2)相比传统的乔利斯基脉冲响应函数, 采用不对变量进入 VAR 模型顺序有依赖的广义脉冲响应函数, 对贵州航空运输能力与当地经 济增长的相互引导方向作分析, 相对更加客观合理。

\section{2. 外部性的机理分析}

贵州地处云贵高原，以山地丘陵地形为主，地势西高东低，地表破碎，区域开发成本大， 不利于公路、铁路和水路基础设施建设，而民航受自然区位因素的阻碍小，因此机场业投资 是贵州交通运输建设的重要环节之一。西部大开发战略实施以来, 贵州的经济发展水平保持 高速增长, 在西南地区发挥出的经济影响力不断增强。滞后的交通基础设施显然会阻碍贵州 经济发展水平的进一步提高, 新出台的《贵州综合交通运输 “十三五” 发展规划》大大强化 了对交通基础设施建设的投资。面对经济新常态，“十三五” 规划提出将对综合交通建设进行 7600 亿元的投资，加大在航空、公路、铁路方面的投入，民航业属于交通运输高端产业，在 
供给侧应当适度超前供给交通基础设施。贵州民航运输业依托大数据构建 “贵州机场云”, 加 速机场业的发展。

经济学告诉我们基础设施具有典型的外部性，因此在分析交通基础设施投资带来的经济 影响时，有必要考虑其自身特有的外部性。跨区域交通基础设施的外部性由市场规模效应和 集聚扩散效应组成。就市场规模效应而言，企业的运输成本随交通基础设施的完善而下降， 当运输成本降低时, 企业更倾向于增加供给, 从而产生正的市场规模效应。集聚扩散效应则 相对复杂，不同地区同一时期或同一地区的不同时期，该效应的正负性都可能存在差异。例 如, 长三角地区的浙江、上海和江苏就共同维持较高的经济增长速度, 交通网络可以将各个 地区的经济活动联系起来，实现相邻区域协同发展。这些在地理位置上靠近的地区利用便利 的交通提高区域合作水平，产生了正向的聚集扩散效应。由于欠发达地区的城市化水平较低、 投资环境较差，方便的交通极可能在欠发达地区产生负向集聚扩散效应，将欠发达地区的资 源禀赋转移到发达地区。贵州经济相对落后, 交通基础设施建设引起的生产要素转移的方向 具有不确定性, 不排除负向集聚扩散效应的可能。当交通基础设施完善带来的负向集聚扩散 效应远大于正向的市场规模效应时，基础设施产生的负外部性显然不利于地区经济发展。

综上，有必要从外部性的视角研究交通运输能力提升对区域经济增长的作用机制，否则 对交通运输投资产生的经济影响的估计都是不可靠的。以下部分将以航空运输业为研究对象, 应用 VAR 模型实证分析贵州航空运输能力与经济发展水平之间的联动关系, 为本文的上述观 点提供依据。

\section{3. 数据来源与说明}

贵州民航运输能力与经济发展水平之间关系，在其运输能力和经济规模的指标上应当有 所体现。本文选取贵州航空运输量(万工作量)和地区生产总值 GDP(十亿人民币)作为指标, 实 证分析航空运输量与地区生产总值之间的定量关系, 进而讨论贵州航空运输能力与经济发展 水平之间的联动机制。为了提高建模和推断的准确性, 本文的样本数据来自西南地区机场生 产统计简报及国家统计局, 搜集了时间跨度从 1998 年第 1 季度至 2017 年第 3 季度的季度数 据，具体数据处理如下:

(1)航空运输量。机场的数量与规模由航空运输投资决定，机场自身的建设水平和民航旅 客吞吐量、货邮吞吐量密切相关。为了综合考虑客运量与货运量, 参照国际机场协会的处理 方法 ${ }^{[9]}$, 令 1 个工作量单位 $=1$ 个旅客 $=0.1$ 吨货物, 则总工作量 $=$ 旅客吞吐量 $+10 \times$ 货邮吞吐量, 本文以万为计量单位并将其记为 AIR。

(2)地区生产总值。地区生产总值在一定程度上可以反映当地的经济发展水平, 因为 GDP 核算时包括了物价变动, 所以本文把 1998 年第 1 季度作为基期, 通过 CPI 对名义 GDP 进行 平减, 进而得到真实的 GDP。

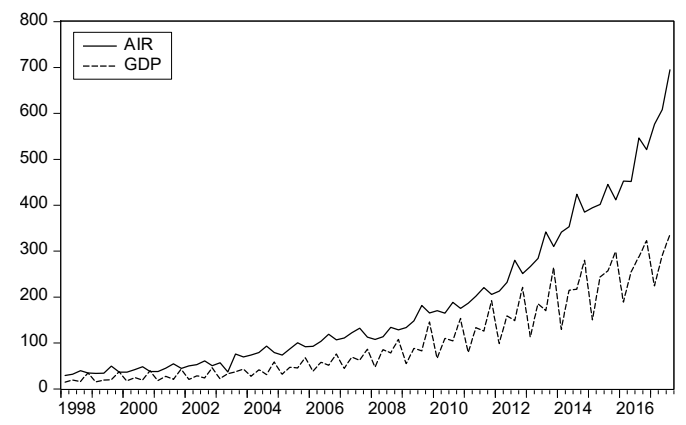

图 1 贵州航空运输量与地区生产总值的时序图

贵州航空运输量与地区生产总值的时序图见图 1。从图 1 中不难发现, 贵州的航空运输 量和国内生产总值存在明显的季节性, 对这两个序列进行 X12 季节调整, 得到的新序列分别 记为 AIR_SA、GDP_SA, 经过季节调整后的序列更加平滑, 在一定程度上降低了季节性特征。 
为了在保持变量之间依存关系的同时, 减轻序列存在的异方差问题, 再对季节调整后的航空 运输量 AIR_SA 和地区生产总值 GDP_SA 取自然对数, 分别用 LAIR 和 LGDP 表示, 序列 LAIR 和 LGDP 大致呈线性增长趋势, 且两者的变动趋势大致相同。以往的研究表明, 经济发展速 度快的地区的航空运输量增长速度一般也较快。因此, 本文进一步计算了航空运输量与地区 生产总值的同比增长率, 两个增长率序列分别记为 RAIR、RGDP, 计算公式如下:

$$
\begin{gathered}
R A I R_{t}=\left(L A I R_{t}-L A I R_{t-4}\right) \times 100 \% \\
R G D P_{t}=\left(L G D P_{t}-L G D P_{t-4}\right) \times 100 \%
\end{gathered}
$$

\section{4. 实证分析}

\section{1 单位根检验}

在建模时, 通过平稳性检验的序列可以避免伪回归问题, 所以本文首先对航空运输量 (LAIR)、地区生产总值(LGDP)、航空运输量增长率(RAIR)以及地区生产总值增长率(RGDP) 进行单位根检验, 检验结果见表 1 。

表 1 各序列的 ADF 检验

\begin{tabular}{c|c|c|c|c|c|c|c}
\hline 变量 & $\begin{array}{c}\text { 检验形式 } \\
(\mathrm{C}, \mathrm{T}, \mathrm{K})\end{array}$ & $\begin{array}{c}\mathrm{ADF} \\
\text { 统计量 }\end{array}$ & 临界值 & 变量 & $\begin{array}{c}\text { 检验形式 } \\
(\mathrm{C}, \mathrm{T}, \mathrm{K})\end{array}$ & $\begin{array}{c}\text { ADF } \\
\text { 统计量 }\end{array}$ & 临界值 \\
\hline LAIR & $(\mathrm{C}, \mathrm{T}, 0)$ & -5.167 & $-4.080^{* * *}$ & $\mathrm{LGDP}$ & $(\mathrm{C}, \mathrm{T}, 2)$ & -2.683 & $-3.162^{*}$ \\
\hline RAIR & $(\mathrm{C}, 0,3)$ & -6.431 & $-3.526^{* * *}$ & RGDP & $(\mathrm{C}, 0,3)$ & -4.386 & $-3.526^{* * *}$ \\
\hline
\end{tabular}

注: ${ }^{* * *} 、{ }^{* *}$ 和 ${ }^{*}$ 分别代表在 $1 \% 、 5 \%$ 和 $10 \%$ 的水平下的临界值, 检验形式里的 $\mathrm{C} 、 \mathrm{~T}$ 和 $\mathrm{K}$ 分别代 表常数项、时间趋势和滞后阶数, 滞后项的阶数由 SC 准则确定。

由表 1 可知, 除序列 LGDP 是 1 阶单整外, 序列 LAIR、RAIR 和 RGDP 均拒绝存在单位 根的原假设。由于原序列 LAIR、LGDP 不是同阶单整序列, 故不再对这两个序列进一步做约 翰森协整检验, 接下来将利用 RAIR、RGDP 序列进行格兰杰因果检验, 展开分析贵州航空运 输量增长率与地区生产总值增长率之间关系。

\section{2 格兰杰因果检验}

表 2 检验了滞后 1 5 阶 RAIR 与 RGDP 之间的格兰杰因果关系。从选取的样本期看, 在 $1 \%$ 的显著性水平下贵州航空运输能力提升与当地经济增长之间存在单向的因果关系, 航空运 输能力提升对经济增长的影响是持续且稳定的, 反之经济增长对航空运输能力提升的作用不 显著，说明贵州的产业经济结构对航空运输的依赖性还较弱。

表 2 RAIR 与 RGDP 的格兰杰因果检验

\begin{tabular}{c|c|c|c}
\hline 滞后阶数 & 零假设 & F 统计量 & $\mathrm{P}$ 值 \\
\hline \multirow{2}{*}{1} & RGDP 不是 RAIR 的格兰杰原因 & 0.137 & 0.713 \\
\cline { 2 - 4 } & RAIR 不是 RGDP 的格兰杰原因 & 14.712 & $3.0 \mathrm{E}-04$ \\
\hline \multirow{2}{*}{2} & RGDP 不是 RAIR 的格兰杰原因 & 0.244 & 0.784 \\
\cline { 2 - 4 } & RAIR 不是 RGDP 的格兰杰原因 & 15.403 & $3.0 \mathrm{E}-06$ \\
\hline \multirow{2}{*}{3} & RGDP 不是 RAIR 的格兰杰原因 & 2.243 & 0.092 \\
\cline { 2 - 4 } & RAIR 不是 RGDP 的格兰杰原因 & 11.204 & $5.0 \mathrm{E}-06$ \\
\hline \multirow{2}{*}{4} & RGDP 不是 RAIR 的格兰杰原因 & 0.633 & 0.641 \\
\cline { 2 - 4 } & RAIR 不是 RGDP 的格兰杰原因 & 8.873 & $1.0 \mathrm{E}-05$ \\
\hline \multirow{2}{*}{5} & RGDP 不是 RAIR 的格兰杰原因 & 0.630 & 0.678 \\
\cline { 2 - 4 } & RAIR 不是 RGDP 的格兰杰原因 & 8.486 & $4.0 \mathrm{E}-06$ \\
\hline
\end{tabular}

\section{3 广义脉冲响应}

因为格兰杰因果检验证实了 RAIR 变动是 RGDP 变动的原因, 所以可以利用 VAR 模型作 
脉冲响应分析, 进一步细化 RAIR 与 RGDP 之间的关系。根据 RAIR、RGDP 序列建立的 VAR 模型滞后阶数评价指标, 可以判断最优滞后阶数为 4 阶。在相应的 AR 根图中, 所有的根都 落于单位圆内, 因此设定的 VAR(4)模型是平稳的。由于传统的乔利斯基脉冲响应的结果严格 依赖于各变量进入 VAR 的顺序, 若将 RAIR 作为第一变量, 则会夸大 RAIR 的影响, 若将 RAIR 作为第二变量, 则会夸小 RAIR 的影响。因此本文放弃了使用乔利斯基脉冲响应函数的 研究方法, 而是使用更为客观合理的, 不对变量排序有依赖的广义脉冲响应函数 GIRF, 展开 讨论 RAIR 与 RGDP 的相互引导方向。图 2 5 分别给出了 VAR(4)模型的广义脉冲响应组图, 冲击的滞后期数取到 20 期(单位: 季度)。

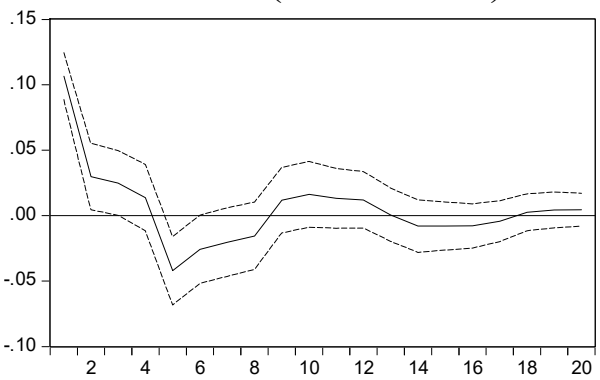

图 2 RAIR 对 RAIR 的广义脉冲响应

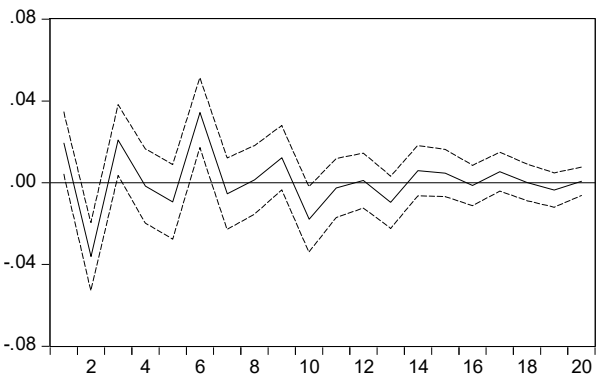

图 4 RGDP 对 RAIR 的广义脉冲响应

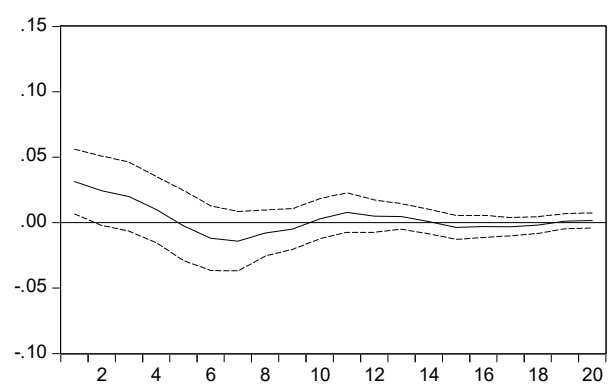

图 3 RAIR 对 RGDP 的广义脉冲响应

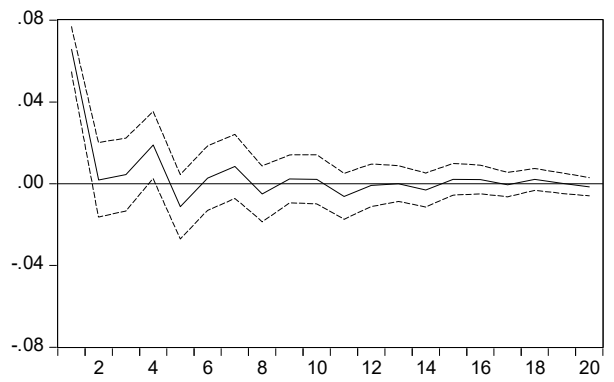

图 5 RGDP 对 RGDP 的广义脉冲响应

图 2 3 给出了 RAIR 对自身和 RGDP 冲击的动态响应过程。由图 2 可知, 给 RAIR 一单 位标准差的正冲击, RAIR 在第 1 期至第 4 期形成正的响应,在第 5 期至第 8 期形成负的响应, 之后又维持了 4 期的正响应, 并逐渐收敛于 0 。图 2 表明, RAIR 的一个正冲击对自身的影响 较大, 在短期产生了一定程度的持续响应, 并呈现以年为单位的周期性波动。由图 3 可知, 给 RGDP 一单位标准差的正冲击, RAIR 呈现震荡收玫并且滞后几期的变化均不明显, 说明 RGDP 对 RAIR 的带动作用较弱, 原因在于影响 RAIR 的因素比较复杂, 航空运输量增长率 还会受到节假日、航空票价等因素影响。

图 4 5 给出了 RGDP 对 RAIR 和自身冲击的动态响应过程。由图 4 可知, 给 RAIR 一单 位标准差的正冲击, RGDP 会呈现明显的上下波动, 说明 RAIR 对 RGDP 的影响较不稳定, 在第 2 期到达负响应的极大值, 在第 6 期到达正响应的极大值, 随后震荡收敛。这表明 RAIR 对 RGDP 的影响显著, 但是持续效应不长。图 4 表明, RAIR 的正向冲击对 RGDP 产生的效 应有正有负，从侧面反映了航空业投资的同时伴随一定程度的资本、人才和技术的外流。该 结果验证了本研究的理论观点, 即贵州航空业发展可能存在负外部性, 但总体而言 RAIR 的 正向冲击有利于 RGDP 的改善。由图 5 可知, 给 RGDP 一单位标准差的正冲击, 对 RGDP 具 有一定持久的正响应, 说明经济增长具有显著的滞后效应。从图 2 5 易见, 长期看来冲击产 生影响最终递减, 说明估计的 $\operatorname{VAR}(4)$ 模型是一个稳定的系统。

\section{4 方差分解}

广义脉冲响应从绝对效果的角度刻画了波动对经济系统的影响, 方差分解则可以从相对 效果的角度评价不同结构冲击的贡献度。表 3 评价了结构冲击在 RAIR、RGDP 动态变化中的 相对贡献。 
表 3 方差分解表

\begin{tabular}{c|c|c|c|c|c|c}
\hline \multirow{2}{*}{ 时期 } & \multicolumn{3}{|c|}{ RAIR 方差分解 } & \multicolumn{3}{c}{ RGDP 方差分解 } \\
\cline { 2 - 7 } & S.E. & RAIR(\%) & RGDP(\%) & S.E. & RAIR(\%) & RGDP(\%) \\
\hline 1 & 0.107 & 100.000 & 0.000 & 0.066 & 8.622 & 91.378 \\
\hline 2 & 0.112 & 97.859 & 2.141 & 0.076 & 29.029 & 70.971 \\
\hline 3 & 0.116 & 96.686 & 3.314 & 0.079 & 33.956 & 66.044 \\
\hline 4 & 0.117 & 96.456 & 3.544 & 0.082 & 31.886 & 68.114 \\
\hline 5 & 0.124 & 96.160 & 3.840 & 0.083 & 32.410 & 67.590 \\
\hline 6 & 0.127 & 96.196 & 3.804 & 0.090 & 42.023 & 57.977 \\
\hline 7 & 0.129 & 95.861 & 4.139 & 0.091 & 41.664 & 58.336 \\
\hline 8 & 0.130 & 95.844 & 4.156 & 0.091 & 41.511 & 58.489 \\
\hline 9 & 0.131 & 95.450 & 4.550 & 0.092 & 42.541 & 57.459 \\
\hline 10 & 0.132 & 95.500 & 4.500 & 0.094 & 44.372 & 55.628 \\
\hline 11 & 0.132 & 95.456 & 4.544 & 0.094 & 44.252 & 55.748 \\
\hline 12 & 0.133 & 95.480 & 4.520 & 0.094 & 44.250 & 55.750 \\
\hline 13 & 0.133 & 95.362 & 4.638 & 0.094 & 44.794 & 55.206 \\
\hline 14 & 0.133 & 95.319 & 4.681 & 0.095 & 44.873 & 55.127 \\
\hline 15 & 0.134 & 95.327 & 4.673 & 0.095 & 45.001 & 54.999 \\
\hline 16 & 0.134 & 95.340 & 4.660 & 0.095 & 44.982 & 55.018 \\
\hline 17 & 0.134 & 95.325 & 4.675 & 0.095 & 45.132 & 54.868 \\
\hline 18 & 0.134 & 95.287 & 4.713 & 0.095 & 45.110 & 54.890 \\
\hline 19 & 0.134 & 95.291 & 4.709 & 0.095 & 45.182 & 54.818 \\
\hline 20 & 0.134 & 95.296 & 4.704 & 0.095 & 45.168 & 54.832 \\
\hline
\end{tabular}

表 3 显示 RAIR 在初期仅受自身波动影响，从第 2 期开始才显现 RGDP 对 RAIR 波动的 冲击(即对预测误差的贡献度), 并且 RGDP 冲击的影响极小, 虽然后期有递增的迹象, 但也 只稳定在 $4.7 \%$ 左右。相对贡献低的原因可能是民航运输量受到经济、政治、机票价格、气候 以及节假日等因素影响, 具有典型的周期性和波动性, 因此经济增长率对航空运输量增长率 的影响并不明显。RGDP 从第 1 期起同时受到自身波动和 RAIR 波动冲击的影响, 随时间推 移, 自身波动的相对影响递减, RAIR 冲击的相对影响递增。RGDP 从第 15 期开始趋于平稳, 稳定在 55\%左右, RAIR 的冲击稳定在 $45 \%$ 左右, 这表明贵州航空运输业能力提升对经济增 长具有较强的推动作用。

\section{5. 结论性评述}

随着国民经济水平的提高，民航的价位从低于一般国民消费水平，到逐渐为大众所接受， 为民航的快速发展创造了条件。就贵州而言, 实证检验的结果表明航空运输能力提升对经济 增长的推动作用远大于经济增长对航空运输的拉动作用, 从经济增长率对航空运输量增长率 的广义脉冲响应在部分时滞期为负可以看出, 短期内贵州航空运输能力提升对地区经济增长 存在一定程度的负外部性。经济增长对航空业的带动效应并不明显, 说明处在经济起飞阶段 的贵州经济对航空运输的依赖性还较弱。因此, 为了进一步提高贵州的开放程度, 不能仅仅 增加机场业的投资, 更应关注民航运输对贵州经济的长远影响, 还需要出台更多的产业政策, 增加当地产业 “粘性”。近年来, 贵州内陆开放实验区、大数据产业示范区和生态文明示范区 的建设将吸引越来越多的企业、资金和人才入驻贵州, 对航空业带来潜在需求。因此, 可以 预期未来建成的 “一枢十六支” 民航机场格局将改善贵州的整体经济结构以及经济增长方式。

\section{致谢}

本文为贵州科学技术基金资助项目《面板数据单位根检验方法及其在 CAPM 中的应用研 究》(黔科合 J 字[2009]2062)、贵州财经大学 2017 年度在校学生科研资助项目的阶段性成果 之一。 


\section{References}

[1] Zhang Jun, Gao Yuan, Fu Yong, Zhang Hong. Why Does China Enjoy So Much Better Physical Infrastructure?[J]. Economic Research Journal,2007(3):4-19.

[2] Guan Xingliang, Lin Xueqin, Hu Shilin, Lu Shasha. Relationship between Transportation System and Urban Spatial Expansion in Wuhan Urban Agglomeration[J]. Progress in Geography,2014,(5):702-712.

[3] Wu Ji-gui, Ye A-zhong. Dynamic Linkages among Transportation, Economic Growth and Carbon Emissions in China: Empirical Research on the Data from 1949 to 2012[J]. Journal of Transportation Systems Engineering and Information Technology,2015,(4):10-17.

[4] Air Transport Action Group. The Economic and Social Benefits of Air Transport 2008[R],2008.

[5] Liu Lanjuan, Dong Wanhao. Empirical Analysis on the Relationship between Civil Aviation Transportation Industry and GDP in China Based on VAR Model[J]. Journal of Finance and Economics,2009,(8):69-78.

[6] Guan Chiming, Ma Qiqi. Empirical Analysis of Dynamic Effect on Economic Growth and Its Mechanism Due to China's Air Transport Investment[J]. China Soft Science,2010,(10):47-56.

[7] Lu Lijuan, Li xiaojin. Relationship among Airspace Resources, Civil Aviation Revenue and National Economy[J]. Shanghai Journal of Economics,2016,(12):20-27.

[8] Zhang Zhancang, Chen Ping, Peng Junjie. The Airport Economy Development of ZAEZ's Innovation on the Regional Development Pattern[J]. Academic Journal of Zhongzhou,2016,(3):17-25.

[9] ACI EUROPE, YORK Consulting. Creating Employment and Prosperity in Europe: An Economic Impact Study Kit[R],2002. 